\title{
Animate and Inanimate Words Demonstrate Equivalent Retrieval Dynamics Despite the Occurrence of the Animacy Advantage
}

\author{
Michael J. Serra* \\ Department of Psychological Sciences, Texas Tech University, Lubbock, TX, United States
}

People demonstrate a memory advantage for animate (living) concepts over inanimate (nonliving) concepts in a variety of memory tasks, including free recall, but we do not know the mechanism(s) that produces this effect. We compared the retrieval dynamics (serial-

OPEN ACCESS

Edited by:

Pietro Spataro,

Mercatorum University, Italy

Reviewed by:

Laura Mieth,

Heinrich Heine University of

Düsseldorf, Germany

Patrick Bonin,

Université de Bourgogne, France

Josefa N. S. Pandeirada,

University of Aveiro, Portugal

Sara Brilhante Félix,

University of Aveiro, Portugal, in

collaboration with reviewer JP

*Correspondence:

Michael J. Serra

michael.serra@ttu.edu

Specialty section:

This article was submitted to

Cognition,

a section of the journal

Frontiers in Psychology

Received: 01 February 2021

Accepted: 05 May 2021

Published: 03 June 2021

Citation:

Serra MJ (2021) Animate and Inanimate Words Demonstrate Equivalent Retrieval Dynamics Despite the Occurrence of the Animacy Advantage.

Front. Psychol. 12:661451. doi: 10.3389/fpsyg.2021.661451 position effects, probability of first recall, output order, categorical clustering, and recall contiguity) of animate and inanimate words in a typical free recall task to help elucidate this effect. Participants were more likely to recall animate than inanimate words, but we found few, if any, differences in retrieval dynamics by word type. The animacy advantage was obtained across serial position, including occurring in both the primacy and recency regions of the lists. Participants were equally likely to recall an animate or inanimate word first on the tests and did not prioritize recalling words of one type earlier in retrieval or demonstrate strong clustering by animacy at recall. Participants showed some greater contiguity of recall for inanimate words, but this outcome ran counter to the animacy effect. Together, the results suggest that the animacy advantage stems from increased item-specific memory strength for animate over inanimate words and is unlikely to stem from intentional or strategic differences in encoding or retrieval by word type, categorical strategies, or differences in temporal organization. Although the present results do not directly support or refute any current explanations for the animacy advantage, we suggest that measures of retrieval dynamics can help to inspire or constrain future accounts for this effect and can be incorporated into relevant hypothesis testing.

\footnotetext{
Keywords: adaptive memory, animacy effect, animacy advantage, free recall, retrieval dynamics, serial position, probability of first retrieval, retrieval contiguity
}

\section{INTRODUCTION}

Across a variety of memory tasks, people often remember more animate (living) concepts than inanimate (nonliving) concepts: the animacy advantage. Most often, researchers examine this effect in the context of the free recall of word lists and have consistently found a recall advantage for animate (e.g., tiger; engineer) over inanimate (e.g., couch; violin) words (e.g., Nairne et al., 2013; Bonin et al., 2014, 2015; Li et al., 2016; Popp and Serra, 2016, 2018; Gelin et al., 2017, 2019; VanArsdall et al., 2017; Leding, 2018, 2019; Meinhardt et al., 2018). The advantage can also occur for recognition (Leding, 2020), nonwords given animate properties 
(VanArsdall et al., 2013), and word pairs (e.g., VanArsdall et al., 2015; DeYoung and Serra, 2021).

We do not know the mechanism(s) that underlies the animacy advantage, but researchers have discredited some potential candidates. The animacy advantage does not seem to occur because animate words are easier to categorize (Gelin et al., 2017; VanArsdall et al., 2017), more mentally arousing (Popp and Serra, 2018), more emotionally arousing (Meinhardt et al., 2018), or more threatening (Leding, 2019, 2020) or invoke greater encoding effort (Bonin et al., 2015; Leding, 2018) than inanimate words. At present, there is conflicting evidence whether animate concepts involve greater visual imagery (i.e., Bonin et al., 2015; Gelin et al., 2019) and conflicting evidence that animate items attract more attention during encoding (i.e., Bonin et al., 2015; Hagen et al., 2018; Bugaiska et al., 2019; Johnson, 2019; Leding, 2019, 2020). Growing evidence suggests the effect might stem from the greater richness of encoding for animate items (e.g., Mieth et al., 2019; Mah et al., 2020; Meinhardt et al., 2020).

The purpose of the present study was not to directly test any previous or new hypotheses for the animacy advantage in memory. Rather, we suggest that comparing the retrieval dynamics (e.g., serial-position effects, probability of first recall, output order, categorical clustering, recall contiguity) of animate vs. inanimate words can help to identify or rule out potential mechanisms for the effect. Most published studies of the animacy advantage in free recall do not report aspects of retrieval dynamics, besides overall recall performance. One exception is Bonin et al. (2015): they found some tendency for participants to recall animate words before inanimate words, and the animacy advantage occurred across serial position in their lists.

The effect of presentation order (serial position) on the free recall of a list of items is one of the oldest documented effects in cognitive psychology (e.g., Robinson and Brown, 1926; Jenkins and Dallenbach, 1927). Compared to words from the middle of the list, participants typically show better memory for words from the beginning (the primacy effect) and better memory for words from the end (the recency effect). Much empirical evidence indicates that these two effects are independent (e.g., Murdock, 1962). For example, discouraging rehearsal during learning only reduces the primacy effect (e.g., Marshall and Werder, 1972; Tan and Ward, 2000), whereas including a distracter task between the last item presented and the test only reduces the recency effect (e.g., Postman and Phillips, 1965; Tan and Ward, 2000). The classic explanation for this independence (e.g., Waugh and Norman, 1965; Glanzer and Cunitz, 1966; Atkinson and Shiffrin, 1968; Milner, 1974; Moscovitch, 1982) is that the primacy effect reflects recall from long-term memory while the recency effect reflects recall from working memory. Although the animacy advantage is typically characterized as an effect of long-term memory (specifically, episodic memory; Nairne et al., 2017; VanArsdall et al., 2017), it can also occur for very short word lists presumably recalled from working memory (cf. Daley et al., 2020). Researchers studying the animacy advantage often design their studies to prevent the primacy effect and recency effects from occurring. For example, Nairne et al. (2013) included buffer words at the start and end of their lists and a distracter task between encoding and recall to deter these effects. In the present experiment, we did not include any buffer words or a distracter task to allow the primacy and recency effects to occur so we could consider if the animacy advantage favors long-term or working memory. Accounts of the animacy advantage that rely on preferential processing for animate over inanimate items might predict a larger animacy effect earlier in the list than later in the list, as it would become more difficult to favor one subset of the words as more words are presented at a fixed rate. In contrast, categorical accounts might predict a greater animacy advantage later in the list than earlier in the list, as the animate and inanimate subsets of words become more apparent.

The first item that participants recall from a list is the basis for the measure known as the probability of first recall (Stefanidi et al., 2018). When participants are not required to recall items in the order studied, they most often recall the firststudied word first when the test is delayed (Howard and Kahana, 1999, 2002) and recall the last-studied word first when the test immediately follows study (Hogan, 1975; Laming, 1999). The first item that participants recall from each list can, therefore, serve as a measure of recency as well as memory strength. A related aspect of retrieval is the output order in which participants recall the words at test. Although some studies concluded that participants recall items with greater memory strength earlier (e.g., Deese and Kaufman, 1957; Postman and Phillips, 1965); others concluded that participants strategically prioritize recalling weaker items sooner to maximize overall recall (e.g., Battig and Slaybaugh, 1969; Brown and Thompson, 1971). For both measures, we might expect first recall to favor animate over inanimate words if they are associated with greater memory strength. If participants are employing a strategy to maximize recall, however, they might recall inanimate words earlier than animate words.

Independent of the output order, participants might show categorical clustering at recall (e.g., recalling items of the same type together), which might reflect a categorical encoding strategy and can aid recall for that category. VanArsdall et al. (2017) disconfirmed this possibility to be a major contributor to the occurrence of the animacy advantage in list recall, but we considered it here for completeness. Those authors concluded that the lack of clustering suggests that the animacy advantage stems more from item-specific processing than a categorical strategy.

The idea of contiguity in retrieval assumes that the recall of one item enhances the recall of another item that occurred in close temporal proximity (i.e., serial position) during encoding (Kahana, 1996). Contiguity can be demonstrated by plotting a conditional response probability curve (CRP; Kahana, 1996; Howard and Kahana, 1999, 2002; Healey et al., 2019). The lag-CRP is typically asymmetric, favoring recall at forward over backward lags but dropping quickly in both directions (Kahana, 1996; Howard and Kahana, 1999, 2002). As our participants expected memory tests, contiguity should occur (cf. Hintzman, 2016). More importantly, we might expect animate items to show greater contiguity than inanimate items, 
as animate items might involve greater temporal organization (cf. Gelin et al., 2017; but see Blunt and VanArsdall, 2021). Lack of a difference in contiguity might instead support the idea that the animacy advantage stems from itemspecific processing.

\section{MATERIALS AND METHODS}

\section{Participants}

In prior studies (e.g., Popp and Serra, 2016, 2018), we obtained rather large animacy effects $\left(\eta_{p}{ }^{2}>0.2\right)$. Various a priori power analyses, therefore, suggest small sample sizes: as few as 15 participants. Given that some of the present analyses only involved portions of the data and some participants have missing values for some measures, we oversampled considerably from that estimate by convenience, to 94 participants. These 94 participants were undergraduate college students enrolled in Introductory Psychology at Texas Tech University. They participated for class credit. Their mean age was 18.91 years old $(S D=1.37)$. Of the participants, 65 reported their gender as female, and 29 reported their gender as male. We did not exclude any participants from the analysis.

\section{Materials}

The materials were 1,097 words (554 animate and 543 inanimate) from which a custom computer program created a random set of animate (e.g., astronaut and chicken) and inanimate (e.g., bicycle and doormat) words for each participant. Two independent raters identified the words as either animate or inanimate ahead of time; we only used words for which they agreed. The animate and inanimate words had a similar mean number of letters (7.42 vs. 7.20 , respectively), valence (5.13 vs. 5.31) and arousal (4.41 vs. 3.99) ratings (Warriner et al., 2013), concreteness ratings (4.26 vs. 4.68; Brysbaert et al., 2014), and age of acquisition (8.24 vs. 7.68; Kuperman et al., 2012). We provide the words, their attributes, and the likelihood of recalling each in the present experiment at https://osf.io/ $\mathrm{rfbc2}$. MANOVA revealed that the animate and inanimate words differed statistically on all but the number of letters (all other $p s<0.01$ ), but each of these variables accounted for less than $1 \%$ of the variance in the likelihood of recall. Most of these differences would have favored the recall of inanimate words, yet we still obtained the animacy advantage (as in earlier experiments, with a $\eta_{\mathrm{p}}^{2}$ around 0.2 ).

\section{Procedure}

In each session, up to five participants completed the task on individual computers running a custom computer program, in the same room, separated by cubicle dividers. Task instructions indicated that the participants would study five lists of words and test over each list but did not mention animacy. Before beginning the task, the computer program randomly selected 50 animate and 50 inanimate words for each participant from the larger set. No word was used more than once per participant. From the 50 animate and 50 inanimate words, the program created five 20-word lists for each participant (each list did not necessarily have 10 animate and 10 inanimate words).

The participants then studied their first list. The computer program presented each word visually on the screen, one at a time, for $5 \mathrm{~s}$ each (with a blank, 250-millisecond inter-item interval). After presenting the last word in the list, the computer program requested that the participants type as many words as they could recall from the list into a field on the computer screen. The participants clicked on a "finished" icon when they could not recall any additional words. This procedure was then repeated for the other four lists. We did not include buffer items at the beginning or end of the lists or a distracter task between study and recall, to allow for the primacy and recency effects.

\section{RESULTS}

We provide the data from the present experiment at https:// osf.io/rfbc2/.

\section{The Animacy Advantage}

We scored recall as either correct or incorrect; we did not award partial credit. For words that the participants spelled incorrectly, we accepted them if they appeared to indicate the correct word phonetically (e.g., accepting "lepard" for "leopard"). We did not award extra points for words recalled more than once in the same list, nor did we penalize or award points for intrusions. We also recorded the output order for the correctly recalled words on each list (if recalled more than once, we used the first output position).

We first considered the main effect of animacy on free recall to ensure that the effect obtained across the five lists and to check for proactive interference (Table 1). Recall performance was greater for animate than inanimate words, $F(1,93)=21.68, M S E=246.97, p<0.001, \eta_{\mathrm{p}}{ }^{2}=0.19$. Recall did not differ across lists, $F(4,372)=1.48, M S E=291.37$, $p=0.207, \eta_{\mathrm{p}}^{2}=0.02$. Animacy and list did not interact, $F(4,372)=1.11, M S E=235.31, p=0.352, \eta_{\mathrm{p}}{ }^{2}=0.01 . \mathrm{We}$, therefore, collapsed the data on list number for the subsequent analyses.

TABLE 1 | Percent recall and output order by animacy and list number.

\begin{tabular}{|c|c|c|c|c|c|c|c|c|}
\hline \multirow[b]{3}{*}{ List \# } & \multicolumn{4}{|c|}{ Percent recall } & \multicolumn{4}{|c|}{ Output order } \\
\hline & \multicolumn{2}{|c|}{ Inanimate } & \multicolumn{2}{|c|}{ Animate } & \multicolumn{2}{|c|}{ Inanimate } & \multicolumn{2}{|c|}{ Animate } \\
\hline & $M$ & $S D$ & $M$ & $S D$ & $M$ & $S D$ & $M$ & $S D$ \\
\hline List 1 & 38.18 & 17.20 & 46.33 & 18.05 & 4.86 & 1.61 & 4.88 & 1.60 \\
\hline List 2 & 41.04 & 20.40 & 46.44 & 18.87 & 5.17 & 1.88 & 5.14 & 1.89 \\
\hline List 3 & 44.48 & 21.07 & 47.76 & 19.96 & 5.13 & 2.03 & 5.34 & 1.86 \\
\hline List 4 & 41.88 & 21.69 & 46.97 & 23.20 & 5.29 & 2.29 & 5.05 & 1.99 \\
\hline List 5 & 41.81 & 22.19 & 43.74 & 22.86 & 5.21 & 2.43 & 5.13 & 2.26 \\
\hline
\end{tabular}

Percent recall is the mean percentage of words of each type that participants correctly recalled on each list. Output order is the mean output order for words of each type that participants correctly recalled on each list. 
For completeness, we also considered intrusions. Participants averaged only $0.45(S D=0.70)$ total inanimate intrusions and $0.34(S D=0.61)$ total animate intrusions after summing across their lists. Intrusions did not differ by animacy, $F(1,93)=1.29$, $M S E=0.41, p=0.260, \eta_{\mathrm{p}}^{2}=0.01$. Given the very low rate of intrusions, we did not consider them further.

\section{Serial-Position Effects}

Figure 1 shows retrieval by animacy across serial position in the lists (collapsed on list number). Some researchers characterize the primacy region as the first three or four items in a list and the recency region as the last four (or more) items in a list (e.g., Stefanidi et al., 2018); others limit these regions to the first two and last two items in each list, respectively (e.g., Kelly and Risko, 2019).

First, we compared participants' recall for animate and inanimate items that appeared in each fifth (quintile) of the lists (Table 2). Recall performance was greater for animate than inanimate words, $F(1,93)=24.60, M S E=232.30, p<0.001$, $\eta_{\mathrm{p}}{ }^{2}=0.21$, and recall differed across quintiles, $F(4,372)=76.66$, $M S E=355.56, p<0.001, \eta_{\mathrm{p}}^{2}=0.45$. Contrasts revealed that recall was greater for items in the first quintile of the lists than for any other quintile (all $p s<0.001$ ), indicating the primacy effect. Similarly, recall was greater for items in the fifth quintile of the lists than for items in the second, third, and fourth quintiles (all $p s<0.001$ ), indicating the recency effect.
Animacy and quintile did not interact, $F(4,372)=0.58$, $M S E=202.59, p=0.679, \eta_{\mathrm{p}}^{2}=0.01$.

Second, we compared participants' recall for animate and inanimate items that appeared in the first two, middle two, and last two positions in the lists (Table 2). Recall performance was greater for animate than inanimate words, $F(1,93)=5.22$, $M S E=626.27, p=0.025, \eta_{\mathrm{p}}{ }^{2}=0.05$, and recall differed by region, $F(2,186)=74.98, M S E=788.09, p<0.001, \eta_{\mathrm{p}}{ }^{2}=0.45$. Contrasts revealed that recall was greater for items in the first region than for items in the middle $(p<0.001)$, indicating the primacy effect, and recall was greater for items in the last region than for items in the middle $(p<0.001)$, indicating the recency effect. Recall was also greater for items in the primacy region than for items in the recency region $(p=0.004)$. Animacy and region did not interact, $F(2,186)=0.38$, $M S E=454.32, p=0.682, \eta_{\mathrm{p}}^{2}<0.01$.

It seems that the animacy advantage is consistent across serial position in lists (cf. Bonin et al., 2015). This outcome suggests that the advantage does not favor either long-term memory or working memory and occurs for both memory systems (cf. Daley et al., 2020). This finding is inconsistent with accounts of the animacy advantage that rely on preferential rehearsal for animate over inanimate items (which might produce a larger animacy advantage earlier in the list) and with categorical-retrieval accounts (which might produce a larger animacy advantage later in the list).

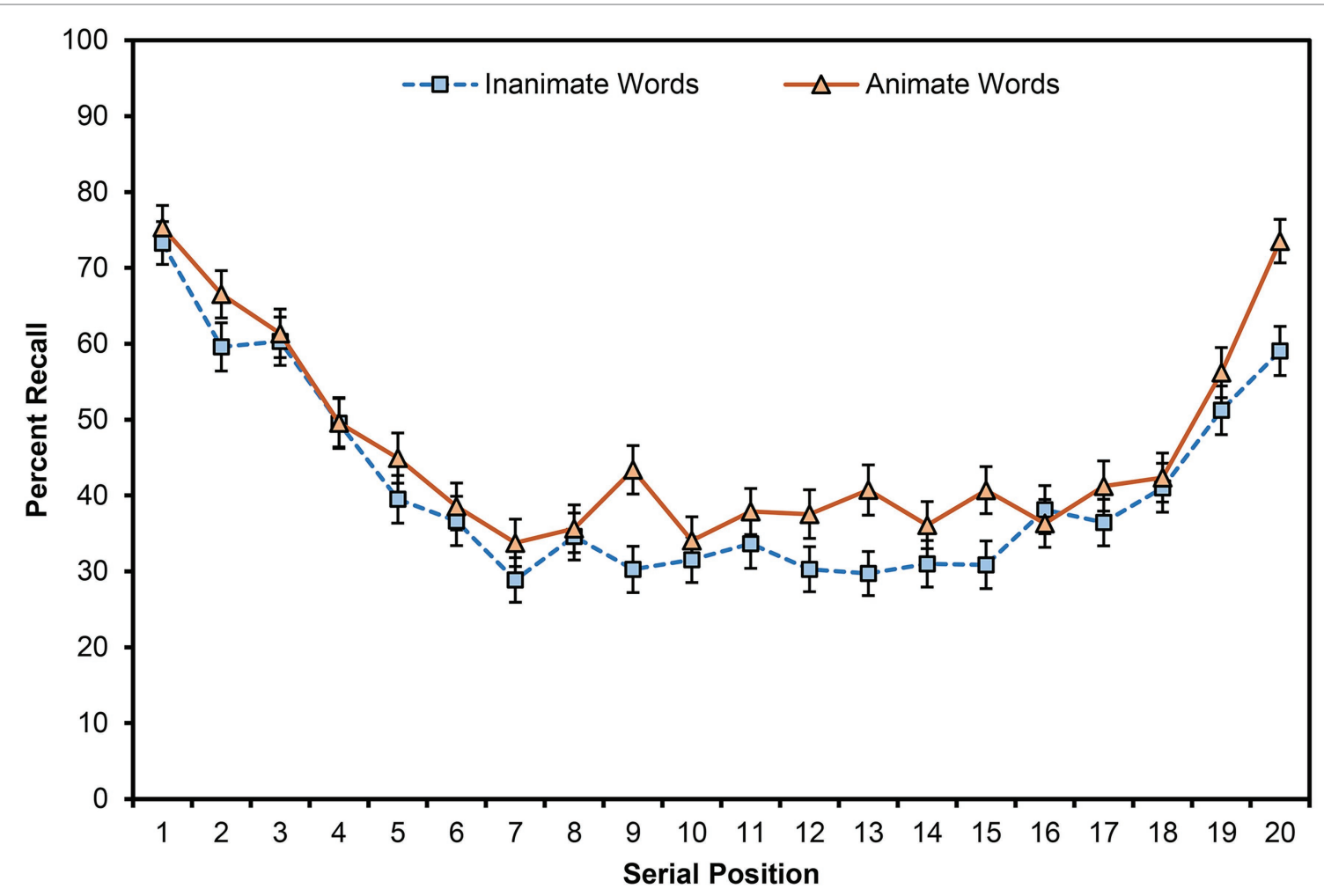

FIGURE 1 | The mean free recall (percent recalled) for animate and inanimate words that were presented at each serial position. Results are collapsed on list number. Error bars are one SEM. 
TABLE 2 | Percent recall and output order by animacy and item grouping.

\begin{tabular}{|c|c|c|c|c|c|c|c|c|}
\hline \multirow[b]{3}{*}{ Item grouping } & \multicolumn{4}{|c|}{ Percent recall } & \multicolumn{4}{|c|}{ Output order } \\
\hline & \multicolumn{2}{|c|}{ Inanimate } & \multicolumn{2}{|c|}{ Animate } & \multicolumn{2}{|c|}{ Inanimate } & \multicolumn{2}{|c|}{ Animate } \\
\hline & $M$ & $S D$ & $M$ & $S D$ & $M$ & $S D$ & $M$ & $S D$ \\
\hline \multicolumn{9}{|c|}{ Even quintile split } \\
\hline Items 1-4 & 60.79 & 21.59 & 63.77 & 23.27 & 4.45 & 1.81 & 4.37 & 1.92 \\
\hline Items 5-8 & 35.05 & 21.94 & 38.60 & 21.36 & 6.57 & 2.05 & 6.15 & 1.88 \\
\hline Items 9-12 & 32.08 & 20.42 & 37.87 & 18.61 & 6.56 & 2.24 & 6.29 & 2.11 \\
\hline Items 13-16 & 32.13 & 18.89 & 38.78 & 21.99 & 6.85 & 2.48 & 6.60 & 2.45 \\
\hline Items 17-20 & 47.47 & 20.10 & 53.15 & 21.45 & 5.13 & 2.80 & 5.12 & 2.99 \\
\hline \multicolumn{9}{|c|}{ Three region split } \\
\hline Items $1 \& 2$ & 67.17 & 25.16 & 71.69 & 26.63 & 4.13 & 2.25 & 3.75 & 2.12 \\
\hline Items 10 \& 11 & 33.69 & 28.16 & 36.74 & 25.16 & 6.95 & 2.53 & 6.44 & 2.38 \\
\hline Items 19 \& 20 & 56.96 & 28.51 & 63.83 & 28.17 & 4.57 & 3.22 & 5.04 & 3.37 \\
\hline
\end{tabular}

Percent recall is the mean percentage of words of each type that participants correctly recalled in each specified range of the lists, collapsed across the five lists. Output order is the mean output order for words of each type that participants correctly recalled in each specified range of the lists, collapsed across the five lists.

\section{Probability of First Recall}

We examined the first word that participants recalled on each test. There was a slight advantage for recalling an animate word (56\% of first words recalled) vs. an inanimate word (44\%), but this difference in proportions was not different from chance $(z=1.16, p=0.245,95 \% C I=45.38$ to $66.23 \%)$. Of all the words recalled first, $30.8 \%$ were the first word in that list and $28.9 \%$ were the last word in that list. Although the last item presented is most often the first word recalled when there is no distracter between study and recall (e.g., Howard and Kahana, 1999, 2002), that tendency did not occur here. A similar pattern emerged when considering animacy. When the first word recalled was inanimate, that word was studied first $33.7 \%$ of the time and last $28.3 \%$ of the time. When the first word recalled was animate, that word was studied first $28.6 \%$ of the time and last $29.4 \%$ of the time. These proportions did not differ by animacy, $\chi^{2}(1,279)=0.69$, $p=0.406$.

Participants were clearly more likely to recall animate than inanimate items (suggesting that the former have greater memory strength) and were most likely to recall the first-studied or last-studied word first on the tests (suggesting that memory strength predicted first recall). Any difference in memory strength by animacy, however, did not result in prioritized first recall for animate words (cf. Deese and Kaufman, 1957; Postman and Phillips, 1965; Stefanidi et al., 2018).

\section{Output Order}

We calculated the mean output order for correctly recalled items. First, we considered output order by animacy across the five lists (Table 1). The output order did not differ by animacy, $F(1,77)=0.07, M S E=1.97, p=0.792, \eta_{\mathrm{p}}^{2}<0.01$, and did not differ across lists, $F(4,308)=1.16, M S E=2.76$, $p=0.329, \eta_{\mathrm{p}}{ }^{2}=0.02$. Animacy and list did not interact, $F(4,308)=0.49, M S E=2.12, p=0.744, \eta_{\mathrm{p}}^{2}<0.01$.

Second, we considered the output order by each quintile of the lists, collapsed on list number (Table 2). The output order did not differ for animate and inanimate words, $F(1,70)=3.40, M S E=2.26, p=0.070, \eta_{\mathrm{p}}{ }^{2}=0.05$. The output order differed by quintile, $F(4,280)=25.03$, $M S E=5.63, p<0.001, \eta_{\mathrm{p}}{ }^{2}=0.26$. Contrasts revealed that the output order was lower for items in the first quintile than for any other quintiles (all $p s<0.001$ ), except for the fifth quintile $(p=0.075)$. Similarly, the output order was lower for items in the fifth quintile than for items in the second, third, or fourth quintile (all $p s<0.001$ ). Animacy and quintile did not interact, $F(4,280)=0.44, M S E=2.21$, $p=0.781, \eta_{\mathrm{p}}^{2}=0.01$.

Third, we considered the output order of items from the first two (primacy), middle two (control), and last two (recency) positions of the lists, collapsed on list number (Table 2). The output order did not differ for animate and inanimate words, $F(1,55)=0.62, M S E=2.58, p=0.435, \eta_{\mathrm{p}}^{2}=0.01$. The output order differed by region, $F(2,110)=22.15, M S E=10.02$, $p<0.001, \eta_{\mathrm{p}}{ }^{2}=0.29$. Contrasts revealed that the output order was lower for items in the primacy region than for items in the middle $(p<0.001)$, and the output order was lower for items in the recency region than for items in the middle $(p<0.001)$. The output order did not differ for items in the primacy and recency regions $(p=0.105)$. Animacy and region did not interact, $F(2,110)=2.36, M S E=3.38, p=0.099$, $\eta_{\mathrm{p}}^{2}=0.04$.

Together, these results suggest that participants were not, purposely or not, likely to recall either animate or inanimate words sooner than later on the tests. Much like the firstrecall data, these outcomes are consistent with the idea that participants recall items with greater memory strength earlier (e.g., Deese and Kaufman, 1957; Postman and Phillips, 1965) in terms of primacy and recency, but inconsistent with any similar expectation that they might recall animate items before inanimate items. Further, these outcomes are inconsistent with the idea that participants might have strategically recalled inanimate items before animate items to maximize overall recall (e.g., Battig and Slaybaugh, 1969; Brown and Thompson, 1971). 


\section{Categorical Clustering}

Ignoring priority, we considered whether participants tended to recall animate and inanimate items together during a recall, which suggests a categorical recall strategy, by calculating absolute Kendall Tau nonparametric correlations between animacy and the output order for each participant on each list. Across the five lists, the mean correlations were 0.23 $(S D=0.20), 0.28(S D=0.20), 0.26(S D=0.22), 0.28(S D=0.21)$, and $0.31(S D=0.25)$, respectively. Although all correlations were above zero (all $p s<0.00$, all Cohen's $d>1.19$ ), the magnitudes only averaged around 0.27 . This result suggests that the participants had some tendency to recall animate and inanimate items together, but it does not suggest a strong categorical recall strategy (cf. VanArsdall et al., 2017).

\section{Recall Contiguity}

We considered participants' contiguity of recall by calculating lag conditional response probability (lag-CRP) curves for animate and inanimate items (Figure 2). For each item recalled (the "focal item"), we calculated the likelihood that the next item recalled was encoded up to five positions (lag) before or after that focal item during encoding (cf. Kahana, 1996; Howard and Kahana, 1999). Although some lags exceeded \pm 5 items, we limited presentation and analysis to these bounds because most items were within this range and it is the norm for reporting CRP curves. These curves were typical: dropping quickly as lag increased in absolute magnitude but favoring forward recall (especially at lag +1) over backward recall.
Probability of recall was greater around inanimate than around animate items, $F(1,93)=15.78, M S E<0.01, p<0.001, \eta_{\mathrm{p}}{ }^{2}=0.15$, and also differed by lag, $F(9,837)=109.73, M S E<0.01, p<0.001$, $\eta_{\mathrm{p}}{ }^{2}=0.54$. Contrasts revealed that the probability of recall at lag +1 was greater than at all other lags (all $p s<0.001$ ). Similarly, the probability of recall at lag -1 was greater than at all other lags $(p s<0.001)$ besides lag $+2(p=0.169)$ and was lower than the probability at lag $+1(p<0.001)$. Animacy and lag interacted, $F(9,837)=3.54, M S E<0.01, p<0.001, \eta_{p}{ }^{2}=0.04$. Paired comparisons indicated that the probability of recall was greater around inanimate than around animate items at lag +1 $(p=0.001)$ and lag $+4(p=0.017)$ but did not differ at the other lags, yielding an interaction. Upon conducting a Bonferroni correction for the 10 comparisons, only the difference at lag +1 would remain significant. Given our large sample size, these two differences could also reflect Type I error.

Although a typical pattern of contiguity was apparent in the present results, animate words did not show greater contiguity than inanimate words, despite being better remembered. This outcome further supports the idea that the animacy advantage stems from item-specific processing but probably not from the greater temporal organization for animate over inanimate words (cf. Blunt and VanArsdall, 2021; but see Gelin et al., 2017).

\section{DISCUSSION}

People tend to remember more animate than inanimate concepts in free recall (e.g., Nairne et al., 2013, 2017; VanArsdall et al., 2013, 2017;

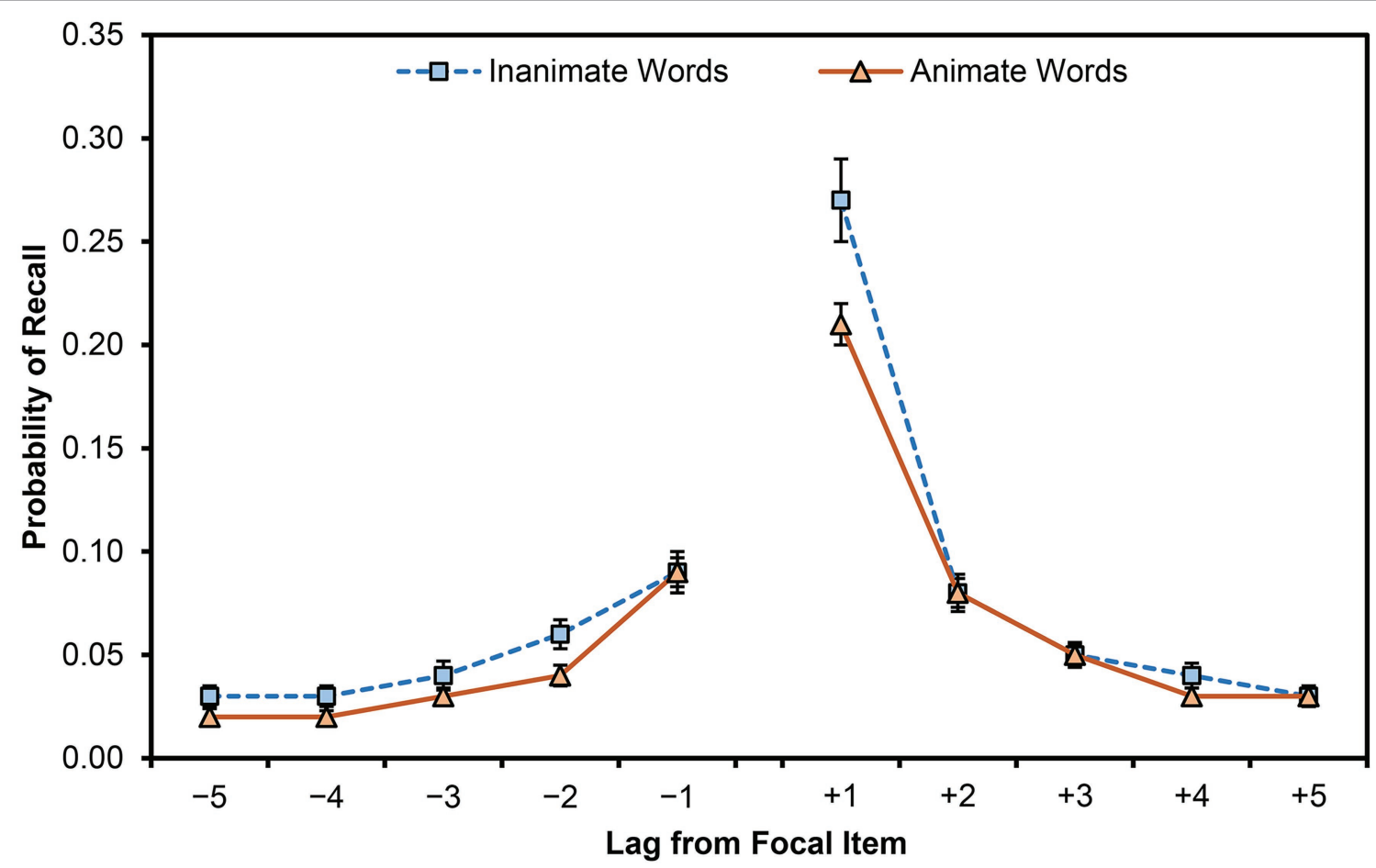

FIGURE 2 | The mean lag conditional response probability (lag-CRP), within \pm 5 lag positions, split by the animacy of the focal word. Results are collapsed on list number. Error bars are one SEM. 
Leding, 2018, 2019), but we do not know what produces this advantage mechanistically. We compared the retrieval dynamics of animate and inanimate words in a typical free recall task (with no buffer words and no distracter task between study and recall) to better understand the nature of this effect. Participants were more likely to recall animate than inanimate words, indicating the animacy advantage, but we found few, if any, differences in retrieval dynamics for animate vs. inanimate words. The animacy advantage was consistent across serial position in the lists, occurring in both the primacy and recency regions (regardless of their operationalization), as well as in the other regions of the lists. Participants did not favor animate over inanimate words in terms of probability of first recall or overall output order, and they also did not demonstrate clustering by animacy at retrieval. Animate words did not demonstrate greater retrieval contiguity than inanimate words (in fact, there was some evidence that inanimate words showed greater contiguity). Overall, the present results suggest that the animacy advantage stems from increased item-specific memory processing for animate over inanimate words (cf. Blunt and VanArsdall, 2021). The effect seems unlikely to stem from intentional differences in encoding or retrieval by animacy or categorical strategies (cf. VanArsdall et al., 2017).

Although the present results do not identify the proximate mechanism(s) that produces the animacy advantage, they can be useful for considering whether a proposed mechanism is feasible or not. We suggest that future tests of proximate mechanisms for the animacy advantage consider the implications of

\section{REFERENCES}

Atkinson, R. C., and Shiffrin, R. M. (1968). "Human memory: a proposed system and its control processes," in Psychology of Learning and Motivation: Advances in Research and Theory. Vol. 2. eds. K. W. Spence and J. T. Spence (New York, NY: Academic Press), 89-195.

Battig, W. F., and Slaybaugh, G. D. (1969). Evidence that priority of free recall of newly learned items is not a recency artefact. J. Verbal Learn. Verbal Behav. 8, 556-558. doi: 10.1016/S0022-5371(69)80104-0

Blunt, J. R., and VanArsdall, J. E. (2021). Animacy and animate imagery improve retention in the method of loci among novice users. Mem. Cogn. doi: 10.3758/s13421-021-01175-0 [Epub ahead of print]

Bonin, P., Gelin, M., and Bugaiska, A. (2014). Animates are better remembered than inanimates: further evidence from word and picture stimuli. Mem. Cogn. 42, 370-382. doi: 10.3758/s13421-013-0368-8

Bonin, P., Gelin, M., Laroche, B., Méot, A., and Bugaiska, A. (2015). The "how" of animacy effects in episodic memory. Exp. Psychol. 62, 371-384. doi: 10.1027/1618-3169/a000308

Brown, S. C., and Thompson, C. P. (1971). Relationship between item strength and order of free recall. J. Verbal Learn. Verbal Behav. 10, 444-448. doi: 10.1016/S0022-5371(71)80044-0

Brysbaert, M., Warriner, A. B., and Kuperman, V. (2014). Concreteness ratings for 40 thousand generally known English word lemmas. Behav. Res. Methods 46, 904-911. doi: 10.3758/s13428-013-0403-5

Bugaiska, A., Grégoire, L., Camblats, A. M., Gelin, M., Méot, A., and Bonin, P. (2019). Animacy and attentional processes: evidence from the Stroop task. Q. J. Exp. Psychol. 72, 882-889. doi: 10.1177/1747021818771514

Daley, M. J., Andrews, G., and Murphy, K. (2020). Animacy effects extend to working memory: results from serial order recall tasks. Memory 28, 157-171. doi: 10.1080/09658211.2019.1699574

Deese, J., and Kaufman, R. A. (1957). Serial effects in recall of unorganized and sequentially organized verbal material. J. Exp. Psychol. 54, 180-187. doi: $10.1037 / \mathrm{h} 0040536$ candidate mechanisms not just for overall recall, but for retrieval dynamics as well. These could be descriptive as in the present study, or studies could take advantage of methods known to reduce or enhance specific aspects of retrieval dynamics and purposely leverage them in tests of a given mechanism. Considering retrieval dynamics in this context can help to inspire, constrain, and test new accounts for the animacy advantage.

\section{DATA AVAILABILITY STATEMENT}

The datasets presented in this study can be found in online repositories. The names of the repository/repositories and accession number(s) can be found at: OSF, https://osf.io/rfbc2/.

\section{ETHICS STATEMENT}

The studies involving human participants were reviewed and approved by Human Research Protection Program, Texas Tech University. The patients/participants provided their written informed consent to participate in this study.

\section{AUTHOR CONTRIBUTIONS}

The author confirms being the sole contributor of this work and has approved it for publication.

DeYoung, C. M., and Serra, M. J. (2021). Judgments of learning reflect the animacy advantage for memory, but not beliefs about the effect. Metacogn. Learn. doi: 10.1007/s11409-021-09264-w

Gelin, M., Bugaiska, A., Méot, A., and Bonin, P. (2017). Are animacy effects in episodic memory independent of encoding instructions? Memory 25, 2-18. doi: 10.1080/09658211.2015.1117643

Gelin, M., Bugaiska, A., Méot, A., Vinter, A., and Bonin, P. (2019). Animacy effects in episodic memory: do imagery processes really play a role? Memory 27, 209-223. doi: 10.1080/09658211.2018.1498108

Glanzer, M., and Cunitz, A. R. (1966). Two storage mechanisms in free recall. J. Verbal Learn. Verbal Behav. 5, 351-360. doi: 10.1016/S0022-5371(66)80044-0

Hagen, T., Espeseth, T., and Laeng, B. (2018). Chasing animals with split attention: are animals prioritized in visual tracking? I-Perception 9, 1-35. doi: $10.1177 / 2041669518795932$

Healey, M. K., Long, N. M., and Kahana, M. J. (2019). Contiguity in episodic memory. Psychon. Bull. Rev. 26, 699-720. doi: 10.3758/s13423-018-1537-3

Hintzman, D. L. (2016). Is memory organized by temporal contiguity? Mem. Cogn. 44, 365-375. doi: 10.3758/s13421-015-0573-8

Hogan, R. M. (1975). Interitem encoding and directed search in free recall. Mem. Cogn. 3, 197-209. doi: 10.3758/BF03212898

Howard, M. W., and Kahana, M. J. (1999). Contextual variability and serial position effects in free recall. J. Exp. Psychol. Learn. Mem. Cogn. 25, 923-941. doi: $10.1037 / / 0278-7393.25 .4 .923$

Howard, M. W., and Kahana, M. J. (2002). A distributed representation of temporal context. J. Math. Psychol. 46, 269-299. doi: 10.1006/jmps.2001.1388

Jenkins, J. G., and Dallenbach, K. M. (1927). The effect of serial position upon recall. Am. J. Psychol. 38, 285-291. doi: 10.2307/1415207

Johnson, J. (2019). Animacy's Effect on Attention and Memory in Humans and Macaques (Macaca mulatta). [master's thesis]. Georgia State University.

Kahana, M. J. (1996). Associative retrieval processes in free recall. Mem. Cogn. 24, 103-109. doi: 10.3758/BF03197276

Kelly, M. O., and Risko, E. F. (2019). Offloading memory: serial position effects. Psychon. Bull. Rev. 26, 1347-1353. doi: 10.3758/s13423-019-01615-8 
Kuperman, V., Stadthagen-Gonzalez, H., and Brysbaert, M. (2012). Age-ofacquisition ratings for 30,000 english words. Behav. Res. Methods 44, 978-990. doi: 10.3758/s13428-012-0210-4

Laming, D. (1999). Testing the idea of distinct storage mechanisms in memory. Int. J. Psychol. 34, 419-426. doi: 10.1080/002075999399774

Leding, J. K. (2018). The animacy advantage in memory: manipulations of levels of processing and survival processing. Am. J. Psychol. 131, 273-281. doi: 10.5406/amerjpsyc.131.3.0273

Leding, J. K. (2019). Adaptive memory: animacy, threat, and attention in free recall. Mem. Cogn. 47, 383-394. doi: 10.3758/s13421-018-0873-x

Leding, J. K. (2020). Animacy and threat in recognition memory. Mem. Cogn. 48, 788-799. doi: 10.3758/s13421-020-01017-5

Li, P., Jia, X., Li, X., and Li, W. (2016). The effect of animacy on metamemory. Mem. Cogn. 44, 696-705. doi: 10.3758/s13421-016-0598-7

Mah, E., Campbell, A., Tamburri, C., Grannon, K., and Lindsay, S. (2020). "A direct replication of Popp and Serra (2016, Experiment 1): better free recall and worse cued recall of animal names than object names" in Poster Presented at the 61st Annual Meeting of the Psychonomic Society, Virtual Meeting; November 19, 2020.

Marshall, P. H., and Werder, P. R. (1972). The effects of the elimination of rehearsal on primacy and recency. J. Verbal Learn. Verbal Behav. 11, 649-653. doi: 10.1016/S0022-5371(72)80049-5

Meinhardt, M. J., Bell, R., Buchner, A., and Röer, J. P. (2018). Adaptive memory: is the animacy effect on memory due to emotional arousal? Psychon. Bull. Rev. 25, 1399-1404. doi: 10.3758/s13423-018-1485-y

Meinhardt, M. J., Bell, R., Buchner, A., and Röer, J. P. (2020). Adaptive memory: is the animacy effect on memory due to richness of encoding? J. Exp. Psychol. Learn. Mem. Cogn. 46, 416-426. doi: 10.1037/xlm0000733

Mieth, L., Röer, J. P., Buchner, A., and Bell, R. (2019). Adaptive memory: enhanced source memory for animate entities. Memory 27, 1034-1042. doi: 10.1080/09658211.2019.1617882

Milner, B. (1974). "Hemispheric specialization: scope and limits," in The Neurosciences: Third Study Program. eds. P. O. Schmitt and F. G. Worden (Cambridge, MA: MIT Press), 75-89.

Moscovitch, M. (1982). "Multiple dissociations of function in amnesia," in Human Memory and Amnesia. ed. L. S. Cermak (Hillsdale, NJ: Erlbaum), 337-370.

Murdock, B. B. Jr. (1962). The serial position effect of free recall. J. Exp. Psychol. 64, 482-488. doi: 10.1037/h0045106

Nairne, J. S., VanArsdall, J. E., and Cogdill, M. (2017). Remembering the living: episodic memory is tuned to animacy. Curr. Dir. Psychol. Sci. 26, 22-27. doi: 10.1177/0963721416667711

Nairne, J. S., VanArsdall, J. E., Pandeirada, J. N., Cogdill, M., and LeBreton, J. M. (2013). Adaptive memory: The mnemonic value of animacy. Psychol. Sci. 24, 2099-2105. doi: 10.1177/0956797613480803
Popp, E. Y., and Serra, M. J. (2016). Adaptive memory: animacy enhances free-recall but impairs cued-recall. J. Exp. Psychol. Learn. Mem. Cogn. 42, 186-201. doi: 10.1037/xlm0000174

Popp, E. Y., and Serra, M. J. (2018). The animacy advantage for free-recall performance is not attributable to greater mental arousal. Memory 26, 89-95. doi: 10.1080/09658211.2017.1326507

Postman, L., and Phillips, L. W. (1965). Short-term temporal changes in free recall. Q. J. Exp. Psychol. 17, 132-138. doi: 10.1080/17470216508416422

Robinson, E. S., and Brown, M. A. (1926). Effect of serial position upon memorization. Am. J. Psychol. 37, 538-552. doi: 10.2307/1414914

Stefanidi, A., Ellis, D. M., and Brewer, G. A. (2018). Free recall dynamics in value-directed remembering. J. Mem. Lang. 100, 18-31. doi: 10.1016/j. jml.2017.11.004

Tan, L., and Ward, G. (2000). A recency-based account of the primacy effect in free recall. J. Exp. Psychol. Learn. Mem. Cogn. 26, 1589-1625. doi: 10.1037//0278-7393.26.6.1589

VanArsdall, J. E., Nairne, J. S., Pandeirada, J. N., and Blunt, J. R. (2013). Adaptive memory. Exp. Psychol. 60, 172-178. doi: 10.1027/1618-3169/ a000186

VanArsdall, J. E., Nairne, J. S., Pandeirada, J. N. S., and Cogdill, M. (2015). Adaptive memory: Animacy effects persist in paired-associate learning. Memory 23, 657-663. doi: 10.1080/09658211.2014.916304

VanArsdall, J. E., Nairne, J. S., Pandeirada, J. N. S., and Cogdill, M. (2017). A categorical recall strategy does not explain animacy effects in episodic memory. Q. J. Exp. Psychol. 70, 761-771. doi: 10.1080/17470218.2016. 1159707

Warriner, A. B., Kuperman, V., and Brysbaert, M. (2013). Norms of valence, arousal, and dominance for 13,915 English lemmas. Behav. Res. Methods 45, 1191-1207. doi: 10.3758/s13428-012-0314-x

Waugh, N. C., and Norman, D. A. (1965). Primary memory. Psychol. Rev. 72, 89-104. doi: 10.1037/h0021797

Conflict of Interest: The author declares that the research was conducted in the absence of any commercial or financial relationships that could be construed as a potential conflict of interest.

Copyright (c) 2021 Serra. This is an open-access article distributed under the terms of the Creative Commons Attribution License (CC BY). The use, distribution or reproduction in other forums is permitted, provided the original author(s) and the copyright owner(s) are credited and that the original publication in this journal is cited, in accordance with accepted academic practice. No use, distribution or reproduction is permitted which does not comply with these terms. 